\title{
Intensified decomposition of vanadium slag via aeration in concentrated
}

\section{$\mathrm{NaOH}$ solution}

\author{
Longjie Liu a,b, Zhonghang Wang a,b, Hao Du a,b, Shili Zheng a, Ulla Lassi c, Yi Zhang a \\ a Key Laboratory of Green Process and Engineering, Institute of Process Engineering, \\ Chinese Academy of Sciences, Beijing 100190, China
}

b University of Chinese Academy of Sciences, Beijing, 100049, China

c University of Oulu, FIN-90014 Oulu, Finland

\begin{abstract}
A new metallurgical process via aeration for the decomposition of vanadium slag in concentrated $\mathrm{NaOH}$ solutionwas proposed. The improvement of oxygen mass transfer coefficient when using aeration at different $\mathrm{NaOH}$ concentration was studied and the effects of critical reaction parameters on vanadium extraction were systematically investigated. The optimal condition was determined to be: alkali concentration of $60 \%$, reaction temperature of $130{ }^{\circ} \mathrm{C}$, alkali-to-ore mass ratio of $6: 1$, stirring speed of $500 \mathrm{rpm}$. The yield of vanadium could reach to $97.41 \%$ after reacting for $6 \mathrm{~h}$ under this reaction condition. The reaction temperature in this new method is $50-270{ }^{\circ} \mathrm{C}$ lower than the current liquid oxidation methods reported in the literatures, and the medium alkaline concentration declined from $85 \%$ to $60 \%$, exhibiting significant advantages in energy consumption as well as reactor design. Kinetics study indicated that the extraction of vanadium was governed by internal diffusion, and the apparent activation energy was calculated to be $17.57 \mathrm{~kJ} / \mathrm{mol}$.
\end{abstract}

\section{Introduction}

Vanadium and its compounds are widely used in many metallurgical industries (B. Liu et al., 2013, H.-B. Liu et al., 2013). There are about 65 types of vanadium-containing minerals in the world and the main raw material for vanadium extraction is titanomagnetite, accounting for about $88 \%$ of vanadium production (Qiu et al., 2011). During steelmaking process using titanomagnetite ores vanadium slag is formed and it is the direct source of vanadium extraction (Wang et al., 2015). The vanadium slag is roasted with $\mathrm{NaCl}, \mathrm{Na} 2 \mathrm{CO} 3$, or $\mathrm{Na} 2 \mathrm{SO} 4$ at $750-850{ }^{\circ} \mathrm{C}$ under an oxidation environment in a rotary kiln or a multiple hearth furnace during the traditional vanadium production process (Voglauer et al., 2004). In order to improve the low vanadium conversion rate of single roasting, multiple roasting processes are applied, leading to the extensive consumption of energy. Furthermore, the sodium salts are decomposed at the high temperature, emitting significant amount of exhausted gases to the environment (B. Liu et al., 2013, H.-B. Liu et al., 2013; Wang et al., 2015). Many improved roasting methods have been developed to improve the vanadium yield and reduce the hazardous residue production by using calcium oxides to replace sodium salts as additives (Li et al., 2011; Li et al., 2016; Yang et al., 2014; Zhang et al., 2015). But the problems such as the low overall resource utilization efficiency, the high-energy consumption and the severe environment pollution remain unsolved. 
Recently, liquid-phase oxidation technologies have been developed to improve the vanadium recovery and ease the environment pollution (B. Liu et al., 2013, H.-B. Liu et al., 2013; Sun et al., 2009; Wang et al., 2015; Zhang et al., 2010). In the new processes, the vanadium slag was treated in either the molten $\mathrm{NaOH}-\mathrm{NaNO} 3$ medium or the highly concentrated alkali metal hydroxide solution (known as the sub-moltenslat medium, SMS) under oxidative conditions. Compared with the roasting method, the liquid-phase oxidation occurred in a pseudo homogeneous media and the mass transfer efficiency is much higher (Wang et al., 2014; Wang et al., 2015). Moreover, fundamental study showed that reactive oxygen species (ROS) generated in the strong alkali liquid media due to the pyrolysis of hydroxyl ions or nitrate ions (Sun et al., 2009; Zhang et al., 2010). The ROS could facilitate the destruction of the mineral structure and intensify the oxidation of the low valence metal oxides in the mineral (Sun et al., 2009; Wang et al., 2015). In the liquid-phase oxidation methods, the vanadium slag can be decomposed at temperatures of $200-400{ }^{\circ} \mathrm{C}$ and the vanadium extraction rate can reach $95 \%$ after reacting for $6 \mathrm{~h}$. Further, no exhausted gas or toxic tailings were discharged during the entire process. However, the high causticity of the reaction medium (above $70 \%$ by weight of $\mathrm{NaOH}$ or $\mathrm{KOH}$ solutions) and high temperature operation condition (above $200{ }^{\circ} \mathrm{C}$ ) caused equipment corrosion and energy consumption (Wang et al., 2015). Therefore, it is of great importance to develop new methods to decrease the reaction temperature and alkali concentration so that the cost for vanadium production using this new process can be lower down.

Oxygen is the main oxidant in liquid oxidation methods, and the increase in oxygen solubility as well as mass transport in the reaction media is therefore critical for the improvement of the reaction efficiency. An improved metallurgical process for chromium extraction was proposed combing the SMS and pressurized leaching (Chen et al.,2013), and it was discovered that under high pressure, the solubility of oxygen in sub-molten solution increased, and consequently the chromium extraction rate.

Aeration is a well-established method to treat wastewater containing organics (Agarwal et al., 2011; Loubière et al., 2004). It was reported that aeration could promote the oxygen dissolution, leading to a significant increase in the air bubble interfacial area and the mass transfer co-efficient (Chern et al., 2001; Terasaka et al., 2011). Aeration can usually be achieved by using rigid nozzles to form a large amount of small bubbles (micro bubbles), and micro bubbles have extremely long stagnation time due to the much decreased buoyancy. Because the bubble surface tension and further the bubble internal pressure, which is the driving force for the oxygen dissolution, the oxygen in micro bubbles usually dissolves fast into the media (Takahashi et al., 2003; Tsang et al., 2004). Most reported applications of micro bubbles for wastewater treatment has been focused on the improvement of gas-liquid mass transfer and contaminant removal effect. It was demonstrated that free radicals generated through collapse of air micro bubbles in the presence of a strong acid (Takahashi et al., 2007). Liu et al. discovered micro bubbles enhanced oxygen transfer rate and contaminant removal in a coagulation floatation process of dyeing wastewater (Liu et al., 2010). Chu et al. discovered that the micro bubbles promoted the ozone mass transfer efficiency and further enhanced the soluble contaminant removal of simulated dyestuff wastewater and practical textile wastewater (Chu et al., 2008). Furthermore, a large number of researchers concluded that micro bubbles could accelerate the formation of the free radicals during the ozonation process (Chu et al., 2007; Li et al., 2009; Takahashi et al., 2007). However, micro bubbles used in hydrometallurgy have rarely 
been reported, especially in the high temperature and strong alkaline solutions like SMS. As in the high alkaline solutions, the traditional micro bubble generator can't resist the corrosion, the applications of the micro bubbles are only limited in the water purification. The goal of this work was therefore to use a pure titanium microporous filter to study the effect of air sparging on the vanadium extraction from vanadium slag. The intensification effects of oxygen mass transfer due to aeration have been discussed, and the ef-fects of various reaction parameters on the vanadium extraction were systematically investigated with reaction kinetics being analyzed. The application of this microporous filter generating microbubbles can also be used in other corrosion solutions both in acid and alkaline solutions.

\section{Materials and method}

\subsection{Minerals}

The vanadium slag samples were obtained from Chengde Iron and Steel Group Co. Ltd., Hebei, China. Prior to the experiments, the sample was treated with ball-milling and drysieved to the diameters between $38 \mu \mathrm{m}$ to $45 \mu \mathrm{m}$. The chemical analysis of a typical sample by ICP-OES was given in Table 1. The reagent sodium hydroxide employed in this work was of analytical grade and purchased from Xilong Chemical Co. Ltd., Commercial pure oxygen produced by Millennium Beijing gas scales center (Beijing) and ultra-pure water obtained from Millipore were used in all experiments.

\subsection{Experimental apparatus and procedure}

All experiments were performed in a $500-\mathrm{mL}$ stainless steel reactor of cylindrical shape under atmospheric pressure with a stainless-steel cover, as illustrated schematically in Fig. 1. The system was equipped with a temperature controller (CKW-2200) with precision of \pm 2 ${ }^{\circ} \mathrm{C}$, a magnetic driven agitator (D-8401WZ) was applied to make the slurry suspended during the experiments. To make up for the loss of water due to evaporation a peristaltic pump (YZ1515X) was used to pump water into the reactor. The oxygen was introduced into the system through a metal microporous filter connected with a gas cylinder, and the oxygen flow rate was controlled by a flow meter.

Before each experiment, the microporous filter was washed with dilute hydrochloric acid and deionized water sequentially to remove the impurities. A predetermined amount of $\mathrm{NaOH}$ and deionized water was added to the reactor under constant stirring and homogeneous medium was formed. The medium was heated to the predetermined temperature and the vanadium slag was added to the reactor, meanwhile the oxygen was continuously introduced using microporous filter. After certain reaction time, about $2 \mathrm{~g}$ of the reacting slurry sample was taken out and diluted using $80 \mathrm{~mL}$ of deionized water, followed by filtration and washing to obtain the residues for further analysis. After the reaction, the slurry was 
diluted with deionized water and then filtered to obtain the leaching solution and leaching cake for further analysis.

The vanadium extraction efficiency from vanadium slag was calculated using the following equation:

$$
\mathrm{X}=\left(1-[\mathrm{C}]_{\mathrm{r}} /[\mathrm{C}]_{0}\right) \times 100 \%
$$

where $[\mathrm{C}] \mathrm{r}$ and $[\mathrm{C}] 0$ were the contents of vanadium in the residues obtained from the reaction stage and that in the original vanadium slag, respectively.

Table 1. The chemical compositions of vanadium slag.

\begin{tabular}{llllllllll}
\hline Component & $\mathrm{V}_{2} \mathrm{O}_{5}$ & $\mathrm{Cr}_{2} \mathrm{O}_{3}$ & $\mathrm{FeO}$ & $\mathrm{MgO}$ & $\mathrm{Al}_{2} \mathrm{O}_{3}$ & $\mathrm{SiO}_{2}$ & $\mathrm{MnO}_{2}$ & $\mathrm{TiO}_{2}$ & $\mathrm{CaO}$ \\
\hline Content, wt.\% & 10.2 & 4.15 & 49.01 & 1.60 & 2.42 & 20.21 & 5.22 & 11.03 & 1.22 \\
\hline
\end{tabular}

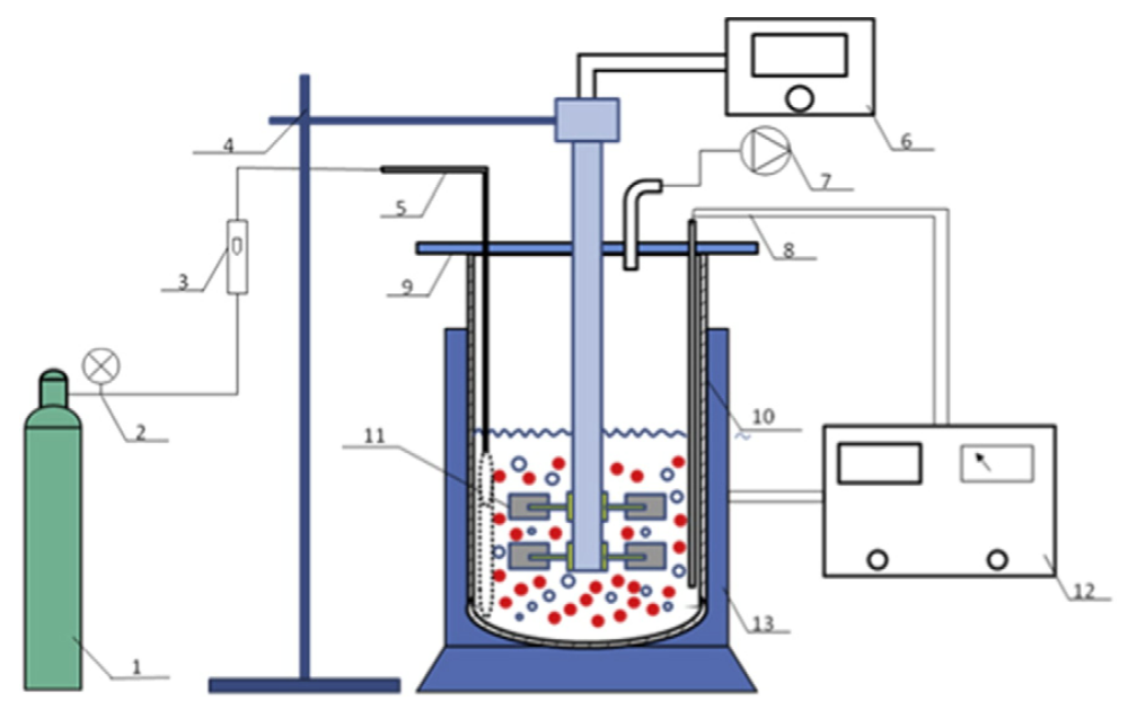

Fig. 1. Schematic diagram of the experimental apparatus in this work. 1. Oxygen cylinder, 2. Oxygen pressure relief valve, 3. Flow meter, 4. Stand, 5. Oxygen introduction pipe with a microporous filter, 6. Stirrer controller, 7. Peristaltic pump, 8. Thermocouple, 9. Cover, 10. Reactor, 11. Push type impeller, 12. Temperature controller, 13. Furnace. 


\subsection{Analysis instruments}

The composition of vanadium slag and residues were analyzed by ICP-OES (PE Optima 6300DV, PerkinElmer). The mineralogical phases of vanadium slag and residues were examined with X-ray diffraction (Empyrean, PANalytical B.V). The SEM-EDS images of the vanadium slag and the residues were obtained with SEM (JSM-7001F +INCA, JEOL) equipment.

\section{Results and discussion}

\subsection{Oxygen mass transfer coefficient in concentrated $\mathrm{NaOH}$}

In the concentrated $\mathrm{NaOH}$ solution, the vanadium slag can be oxidized to produce water soluble sodium vanadate in the presence of oxygen, as shown below.

$$
\mathrm{FeO} \cdot \mathrm{V}_{2} \mathrm{O}_{3}+6 \mathrm{NaOH}+\frac{5}{4} \mathrm{O}_{2} \rightarrow \frac{1}{2} \mathrm{Fe}_{2} \mathrm{O}_{3}+3 \mathrm{H}_{2} \mathrm{O}+2 \mathrm{Na}_{3} \mathrm{VO}_{4}
$$

From the above equation, it is indicated that the improvement of oxygen dissolution can accelerate the vanadium extraction. To investigate the oxygen mass transfer under aeration condition, the classic model for the analysis of surface aerators using the standard unsteady state aeration test was selected, and described as follows (Chern et al., 2001):

$$
\frac{\mathrm{d} C}{\mathrm{dt}}=\mathrm{K}_{L} a\left(\mathrm{C}_{r}^{*}-\mathrm{C}_{0}\right)
$$

where $\mathrm{C}$ is the dissolved oxygen concentration at time $\mathrm{t}, \mathrm{C} \cdot \mathrm{r}$ is the saturated oxygen concentration and KLa the volumetric mass-transfer coefficient for oxygen. Eq. (3) can be readily integrated to yield the following expression for $\mathrm{C}$ as a function of time as shown in Eq. (4) (ASCE, 1997).

$$
\mathrm{C}=\mathrm{C}_{\mathrm{r}}^{*}-\left(\mathrm{C}_{\mathrm{r}}^{*}-\mathrm{C}_{0}\right) \exp \left(-\mathrm{K}_{\mathrm{L}} \text { at }\right)
$$

where $\mathrm{C}_{0}$ is the original dissolved oxygen concentration at $\mathrm{t}=0$. The nonlinear regression analysis based on the Gauss-Newton method is recommended by ASCE to fit equation to the experimental data to calculate the KLa. 
The concentration of dissolved oxygen in the solution was determined by an oxygen solubility detector made by Hanna Instrument (HI98186), and the oxygen flow was determined by the flow meter.

The $\mathrm{KLa}$ as a function of the alkali concentration was studied at the temperature of $30^{\circ} \mathrm{C}$ with the oxygen flow rate of $1 \mathrm{~L} / \mathrm{min}$, and the results were shown in Table 2 . It was observed from Table 2 that with the increase in alkali concentration, the oxygen mass transfer coefficient decreased, which is due to the significant increase in the solution viscosity increased with alkali concentration. Also, the microporous filter used can improve the oxygen mass transfer coefficient. From 0 to $20 \%$, the oxygen mass transfer with microporous filter is three times compared with the oxygen mass transfer with regular tube. While from $30 \%-$ $35 \%$, the oxygen mass transfer is almost 4 times.

Oxygen transfer is a critical part of wastewater treatment process and accounts of as much as $60 \%$ of energy consumption in the activated sludge process (Bangalore and Bellmer, 2006). The improvement in oxygen transfer process can decrease the electrical energy in the treatment process and save a significant capital cost. The effects of impurities in wastewater on the oxygen transfer rate have been studied. Koide et al. (Koide et al., 1976) reported that the volumetric mass-transfer coefficient decreased in the presence of surfactants in water. Gurol and Nekouinaini (Gurol and Nekouinaini, 1985) found that the volumetric mass-transfer coefficient decrease with the surface-active compound and increase with the alcohol and carboxylic acid. Chern et al. (Chern et al., 2001) reported that the addition of the impurities can reduce the oxygen transfer rate in the gas bubble zone while surfactant and diatomaceous earth enhance the oxygen transfer rate in surface reaeration zone. All the studies in the literatures used the ASCE oxygen mass transfer model to evaluate the effect of impurities on the volumetric mass-transfer coefficient. In this study, based on the ASCE analysis, we can draw the conclusion that the microporous filter used in the system can greatly improve the oxygen mass transfer, especially in the high alkaline concentration solution.

Table 2 Effect of the $\mathrm{NaOH}$ concentration on oxygen mass transfer.

\begin{tabular}{lllll}
\hline NaOH concentration (\%) & \multicolumn{5}{l}{ Oxygen mass transfer $\left(\mathrm{h}^{-1}\right)$} \\
\cline { 2 - 5 } & 0 & 20 & 30 & 35 \\
\hline With regular tube & 89.64 & 54.79 & 14.58 & 8.31 \\
With microporous filter & 212.04 & 165.02 & 70.74 & 38.12 \\
\hline
\end{tabular}

\subsection{Decomposition of vanadium slag with aeration under different conditions}

\subsubsection{Effect of microporous filter on vanadium extraction}

The influence of aeration on the extraction process was first examined under the following conditions: $\mathrm{NaOH}$ solution concentration $60 \mathrm{wt} \%$, reaction temperature $130^{\circ} \mathrm{C}$, alkali to ore mass ratio of $6: 1$, oxygen flow rate of $1.0 \mathrm{~L} / \mathrm{min}$, stirring speed of $500 \mathrm{rpm}$, ore particle size 
is between $38 \mu \mathrm{m}$ to $45 \mu \mathrm{m}$, and the reaction time of $6 \mathrm{~h}$. The extraction of vanadium using microporous filter with different pore size from 20 to $100 \mu \mathrm{m}$ and regular tuber (diameter 6 $\mathrm{mm}$ ) was summarized in Fig. 2.

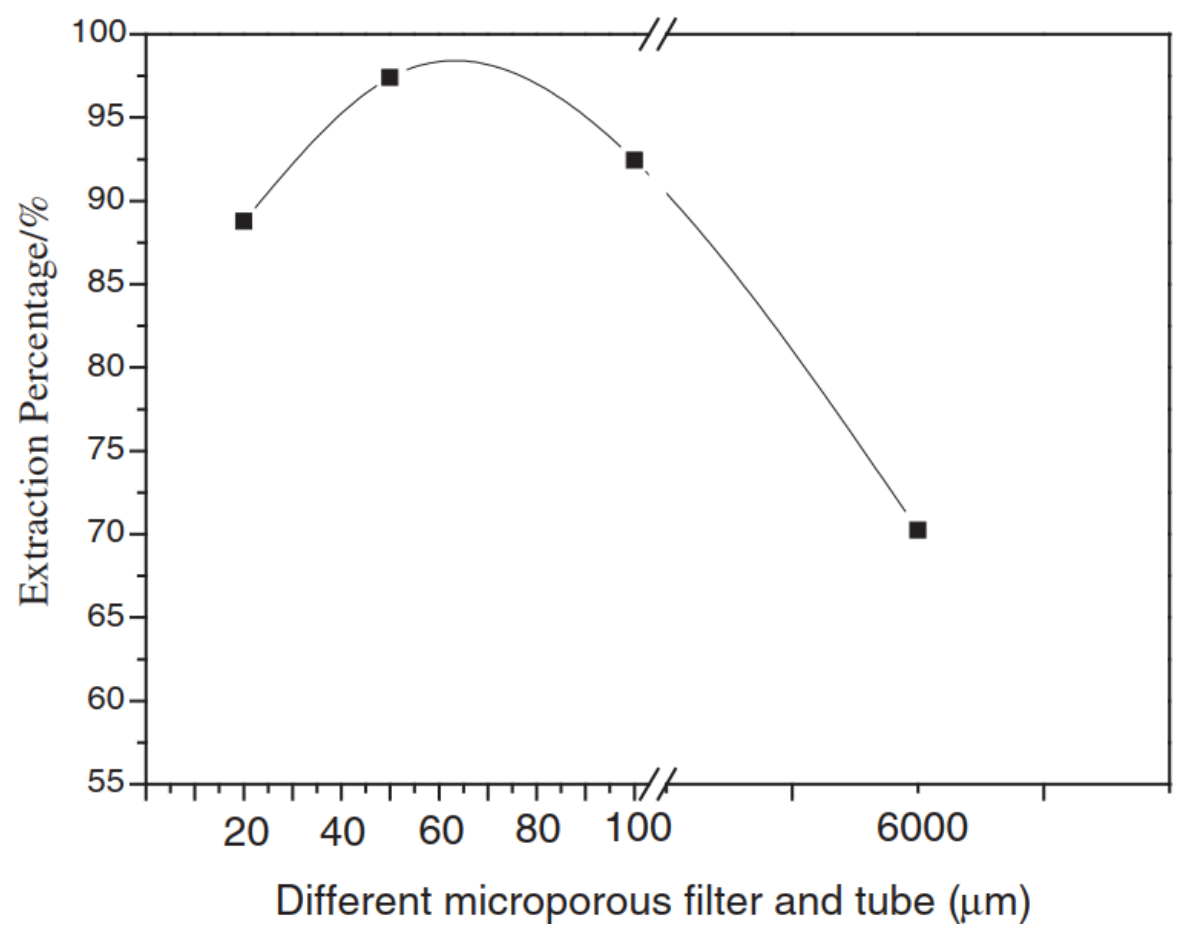

Fig. 2. Effect of the different microporous filter on the extraction of vanadium after $6 \mathrm{~h}$.

As shown in Fig. 2, the extraction percentage of vanadium increased significantly when the microporous filter was used, moreover, the vanadium extraction percentage increased when the pore size of the microporous filter is decreased from $100 \mu \mathrm{m}$ to $50 \mu \mathrm{m}$, this is due to the reason that the micro bubbles generated by the microporous is smaller with the $50 \mu \mathrm{m}$ microfilter.(Burns et al., 1997) However, when the pore size of the microporous filter is 20 $\mu \mathrm{m}$, the vanadium extraction is lower, this is caused by the vanadium slag particles (38-45 $\mu \mathrm{m}$ ) that can block the pore, which decrease the oxygen dissolution. Thus, the pore size of $50 \mu \mathrm{m}$ microporous filter was selected.

Enhanced by the self-compression and shrinking of bubbles, the oxygen micro bubbles diffuse into reaction solutions through the interface. As the inside of the gas pressure is increased with the decreased of the bubbles $(\Delta P=4 \sigma / d, \Delta P$ is the different pressure between the inside and outside of a bubble, ois the surface tension, $d$ is the spherical bubble diameter), the oxygen mass transfer is much quicker (Takahashi et al., 2003; Tsang et al., 2004). Moreover, the micro bubbles are proved to have large interfacial area concentration per unit gas volume and low relative velocity between the bubbles and liquid phase (Maeda et al., 2015), which therefore improve the efficiency of the vanadium oxidation. 


\subsubsection{Effect of alkali concentration}

The influence of alkali concentration on the extraction process in aeration system was studied under the following conditions: reaction temperature $130^{\circ} \mathrm{C}$, alkali to ore mass ratio of $6: 1$, oxygen flow rate of $1.0 \mathrm{~L} / \mathrm{min}$, stirring speed of $500 \mathrm{rpm}$, ore particle size between 38 $\mu \mathrm{m}$ to $45 \mu \mathrm{m}$, and the reaction time of $6 \mathrm{~h}$. The extraction of vanadium over alkali concentrations with time were summarized in Fig. 3.

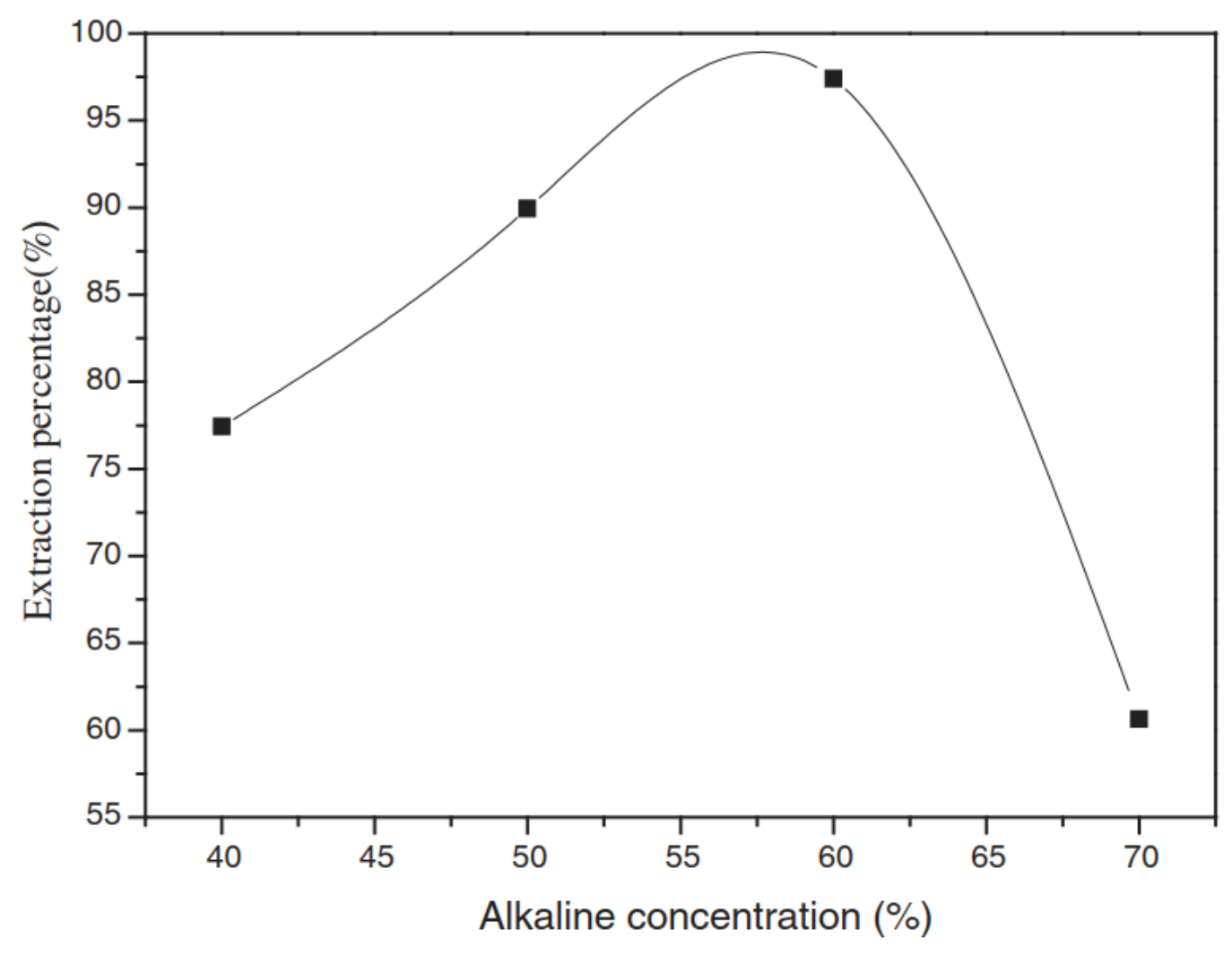

Fig. 3. Effect of the alkali concentration on the extraction of vanadium after $6 \mathrm{~h}$.

From the results shown in Fig. 3 , it is observed that when the alkali concentration increased from $40 \mathrm{wt} \%$ to $60 \mathrm{wt} \%$ the extraction percentage of vanadium significant increased. The reason for this is that the reaction activity of dissolved oxygen and the hydroxide ions increases with alkali concentration goes up and thus the leaching speed is accelerated (Pitzer, 1973). However, when the alkali concentration further increased from $60 \mathrm{wt} \%$ to 70 $w t \%$, the extraction of vanadium decreased, this is due to the dramatic decrease in mass transfer efficiency and the oxygen solubility when the alkaline concentration is too high at low temperature (Mishima et al., 1996; Mishima et al., 1997), which is proved by our oxygen transfer efficiency study. Thus, the alkali concentration of $60 \mathrm{wt} \%$ was selected. 


\subsubsection{Effect of reaction temperature}

The effect of reaction temperature on the vanadium extraction process in aeration system was studied in $60 \mathrm{wt} \% \mathrm{NaOH}$ solution with an alkali to ore mass ratio of $6: 1$, an oxygen flow rate of $1.0 \mathrm{~L} / \mathrm{min}$, a stirring speed of $500 \mathrm{rpm}$, an ore particle size between $38 \mu \mathrm{m}$ to $45 \mu \mathrm{m}$, and the reaction time to be $6 \mathrm{~h}$. The results were presented in Fig. 4 .

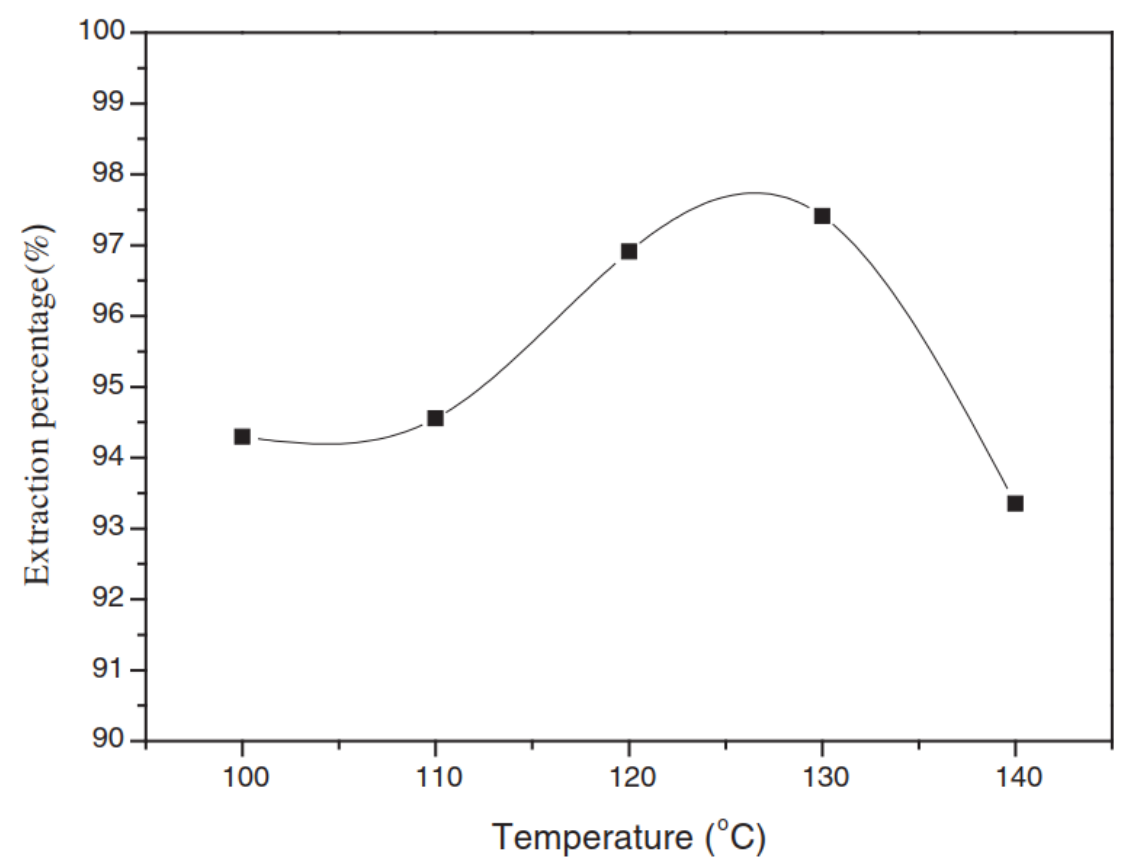

Fig. 4. Effect of the reaction temperature on the extraction of vanadium after $6 \mathrm{~h}$.

From Fig. 4 it can be shown that the extraction of vanadium increased as the temperature increased from $100^{\circ} \mathrm{C}$ to $130^{\circ} \mathrm{C}$, the reason for this is that higher temperature speeded up the oxygen mass transfer for the decrease in medium viscosity. The boiling point of the 60 wt\% alkaline solution is about $140{ }^{\circ} \mathrm{C}$ and it is not safe to operate at the boiling point condition in industry process. As a result, the temperature of $130^{\circ} \mathrm{C}$ was chosen for further experiments.

\subsubsection{Effect of alkali to ore mass ratio}

The influence of alkali to ore mass ratio on the extraction rate of vanadium was investigated at temperature of $130{ }^{\circ} \mathrm{C}$ under the conditions of alkali concentration $60 \mathrm{wt} \%$, oxygen flow $1.0 \mathrm{~L} / \mathrm{min}$, stirring speed $500 \mathrm{rpm}$, and the ore particle size between $38 \mu \mathrm{m}$ to $45 \mu \mathrm{m}$. The results were presented in Fig. 5. 


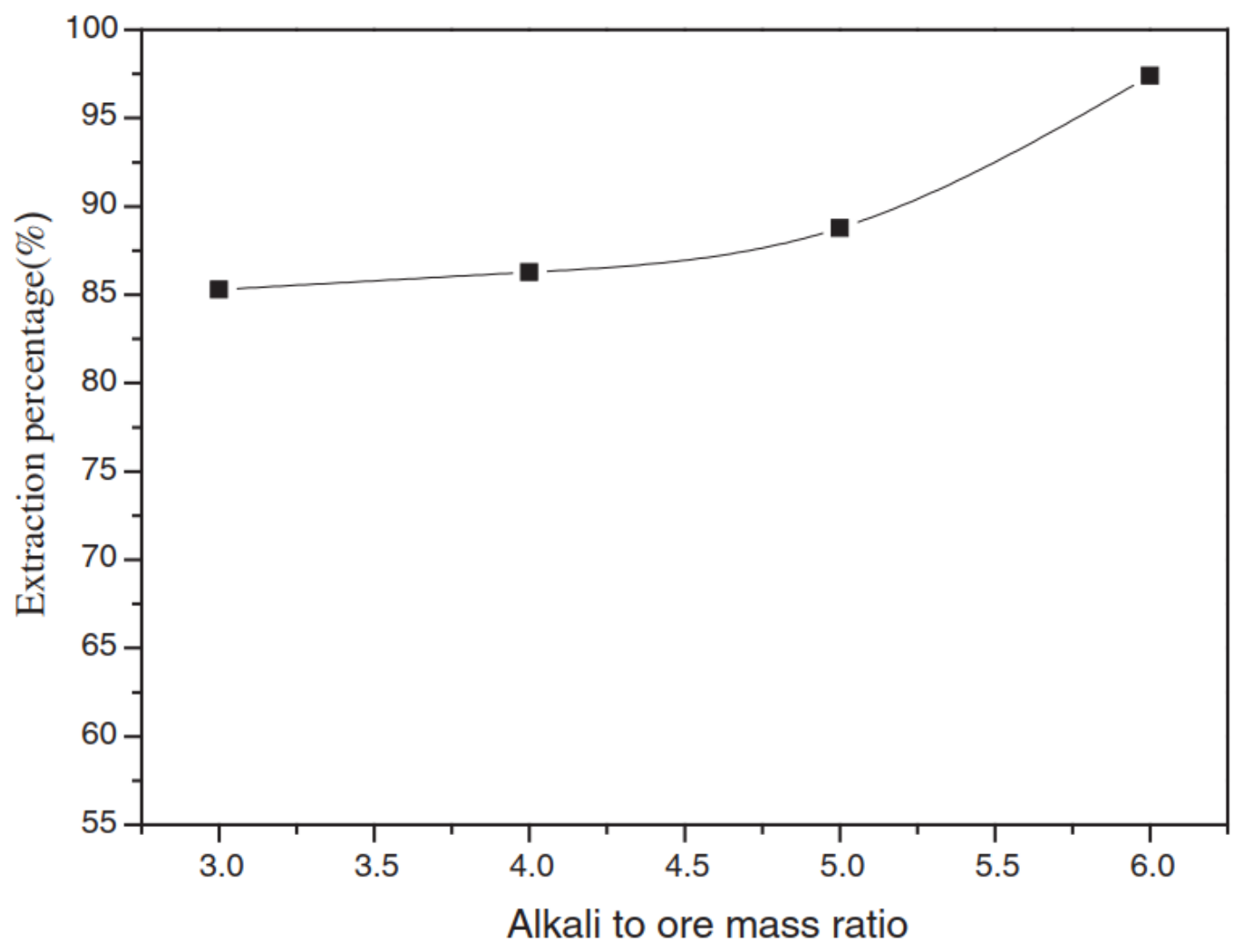

Fig. 5. Effect of alkali to ore mass ratio on the extraction of vanadium after $6 \mathrm{~h}$.

As shown from Fig. 5, the extraction of vanadium increased dramatically with the alkali to ore mass ratio increased from $3: 1$ to $6: 1$, which is due to the mass transfer efficiency improvement and the minerals effectively mixed with the reaction medium with less vanadium slag. Thus, an alkali to ore mass ratio of $6: 1$ was selected.

\subsubsection{Effect of agitation}

The impact of agitation was studied in $60 \mathrm{wt} \% \mathrm{NaOH}$ solutions at $130{ }^{\circ} \mathrm{C}$ with an alkali to ore ratio of $6: 1$, oxygen flow rate of $1 \mathrm{~L} / \mathrm{min}$, and the ore particle size between $38 \mu \mathrm{m}$ to 45 $\mu \mathrm{m}$, and reaction time of $6 \mathrm{~h}$. The results were presented in Fig. 6 . When the stirring speed was low, the mineral particles could not be sufficient for the mix of the oxygen and the solution, and the increase in agitation could promote the extraction speed. However, as the agitation was enough to overcome the mass transfer barrier for the reaction, further increase in the stirring speed is not necessary. Thus, a stirring speed of $500 \mathrm{rpm}$ was selected.

\subsubsection{Summary}

The extraction of vanadium showed an analogously parabolic characteristic with the alkali concentration and reaction temperature increased, and the increase in the alkali to ore mass ratio and stirring speed promoted the extraction process. The optimal reaction conditions 
was determined to be alkali concentration of $60 \mathrm{wt} \%$, reaction temperature of $130{ }^{\circ} \mathrm{C}$, alkali to ore mass ratio of $6: 1$, stirring speed of $500 \mathrm{rpm}$, and the extraction percentages of vanadium can reach $97.41 \%$ after reacting for $6 \mathrm{~h}$.

Table 3 summarized the comparison of the aeration method with liquid-phase oxidation methods reported in the literatures. We can see that the reaction medium concentration and temperature in this new method were much better than those of reported in the literatures.

The process we presented here would be much easier to operate in practical application with lower temperature and alkali concentration, and the equipment corrosion problem could also be significantly alleviated.

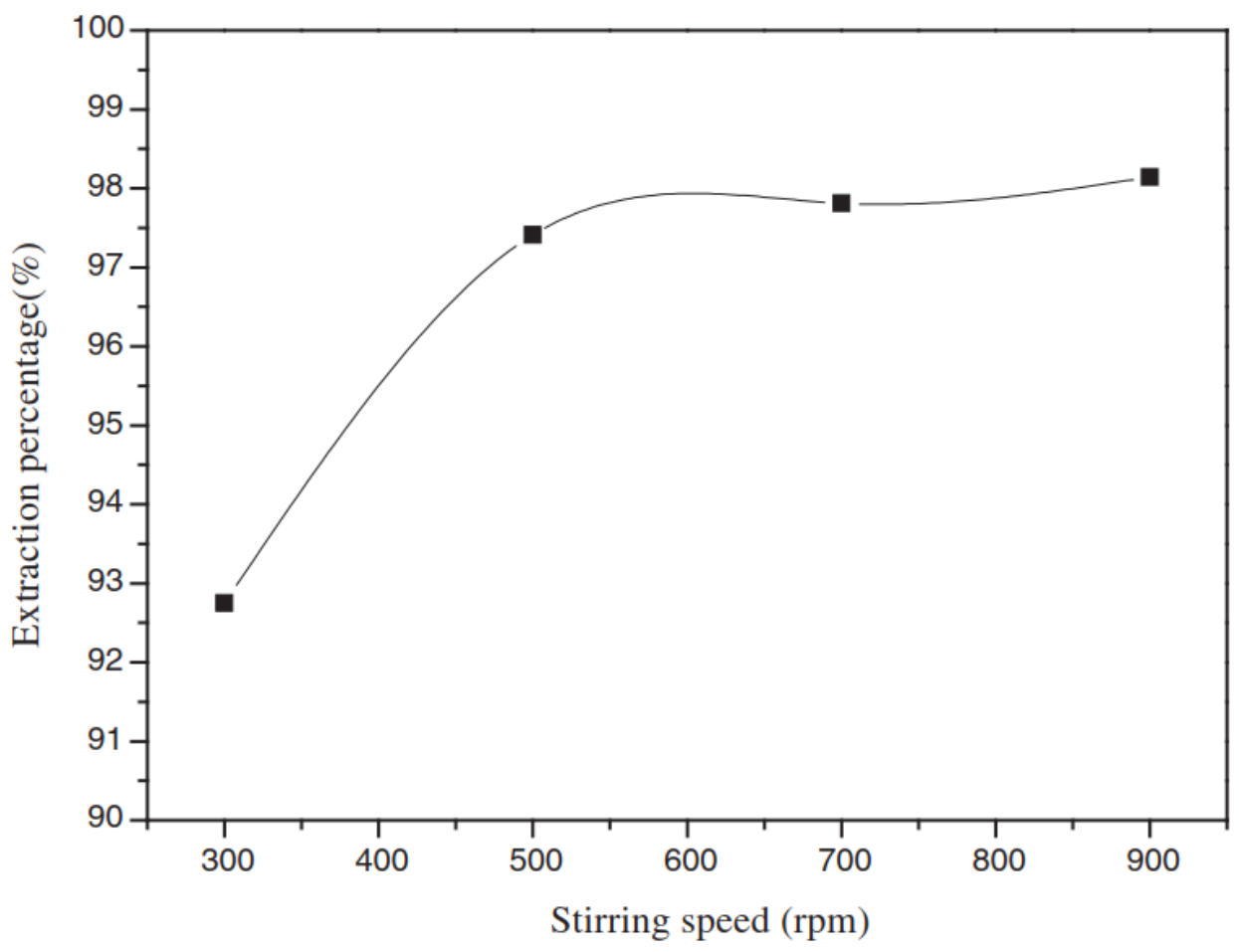

Fig. 6. Effect of stirring speed on the extraction of vanadium after $6 \mathrm{~h}$. 
Table 3. Parameter comparison of typical liquid phase oxidation methods.

\begin{tabular}{|c|c|c|c|c|c|}
\hline Media $^{a}$ & $\mathrm{~T}^{\mathrm{b}}\left({ }^{\circ} \mathrm{C}\right)$ & $C^{c}$ & $\mathrm{ATO}^{\mathrm{d}}$ & Time (min) & $X^{e}(\%)$ \\
\hline $\mathrm{NaOH}-\mathrm{NaNO}_{3}$ MS (Liu et al., 2012) & 400 & 100 & $2: 1$ & 360 & 93.7 \\
\hline NaOH SMS (Wang Dawei) & 210 & 80 & $4: 1$ & 360 & 95 \\
\hline KOH SMS (H.-B. Liu et al., 2013) & 180 & 75 & 4:1 & 300 & 95 \\
\hline This work & 130 & 60 & $6: 1$ & 360 & 97 \\
\hline
\end{tabular}

${ }^{a}$ MS and SMS represent molten salt and sub-molten salt, respectively.

b $\mathrm{T}$ represents temperature.

c $C$ represents the medium concentration.

d ATO represents the alkali to ore mass ratio.

e $\mathrm{X}$ represents the extraction of vanadium.

\subsection{Kinetics analysis}

The apparent activation energy and the reaction rate constant can be calculated with the influence of temperature on vanadium slag decomposition. It is useful for reactor scale-up and project exercise to know the apparent activation energy and the reaction rate constant. Moreover, the decomposition mechanisms can be proposed according to this calculation.

In order to understand the micro-structure of the reacted vanadium slag, the polished surface of the leaching residue was prepared and examined. The back scattered electron image and the related EDS results of the residue's cross-section were given in Fig. 7 and Table 4. The EDS results showed that the central part of the particle mainly consisted of $\mathrm{Cr}$, $\mathrm{V}, \mathrm{Fe}, \mathrm{Mg}$ and $\mathrm{O}$, while in the outer-ring $\mathrm{B}$ the percentage of vanadium was $0.38 \%$, which was the reacted vanadium slag. At point $C$, the chromium remained to be $1.31 \%$, suggesting that the chromium in the surface layer of the vanadium slag was not reacted thoroughly. The compositional change of the cross-section indicted that the solid product formed an ash layer and became thicker leaching process proceeded.

The existence of the product layer had a strong effect on the reaction process, and it was proposed that the vanadium dissolution process should be controlled by diffusion in the reaction product layer(Sun et al., 2009).

Based on the former studies and the above analysis, we can draw the conclusion that the alkaline leaching process of vanadium ore mostly followed the shrinking core model (B. Liu et al., 2013; Wang et al., 2015). The reaction rate can be generally controlled by the following step: diffusion of reactants through the medium (external diffusion) diffusion of reactants through the ash layer (internal diffusion), or chemical reaction at the surface of the core of un-reacted materials (surface reaction).

If the vanadium ore particles have a spherical geometry and the process is controlled by external diffusion, the shrinking core model can be described as follows: 


$$
\mathrm{x}=\mathrm{kt}
$$

When the surface reaction is the controlling step, the kinetics of the process is simplified as follows:

$$
1-(1-\mathrm{x})^{1 / 3}=\mathrm{k}_{\mathrm{r}} \mathrm{t}
$$

If the internal diffusion is the controlling step, the kinetics of the process should be calculated by the following equation:

$$
1+2(1-\mathrm{x})-3(1-\mathrm{x})^{2} / 3=\mathrm{k}_{\mathrm{d}} \mathrm{t}
$$

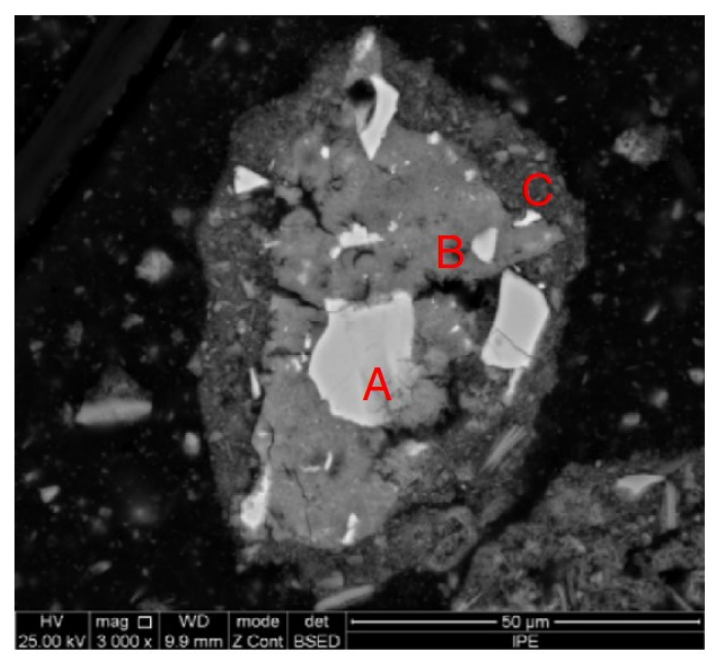

Fig. 7. The back scattered electron image of the residue after reacting for $6 \mathrm{~h}$ 
Table 4. The compositions of the results determined on the cross-section of the residue.

\begin{tabular}{llll}
\hline & \multicolumn{3}{l}{ Element percentage (\%) } \\
\cline { 2 - 4 } Element & $\mathrm{A}$ & $\mathrm{B}$ & $\mathrm{C}$ \\
\hline $\mathrm{O}$ & 25.04 & 32.09 & 31.55 \\
$\mathrm{Na}$ & - & 1.96 & 1.65 \\
$\mathrm{Mg}$ & 1.04 & 3.58 & 2.17 \\
$\mathrm{Al}$ & 1.56 & 0.68 & 3.56 \\
$\mathrm{Si}$ & 0.51 & 10.33 & 7.35 \\
$\mathrm{Ca}$ & - & 1.42 & 1.8 \\
$\mathrm{Ti}$ & 11.4 & 4.18 & 7.41 \\
$\mathrm{~V}$ & 15.8 & 0.38 & 0.18 \\
$\mathrm{Cr}$ & 4.61 & - & 1.31 \\
$\mathrm{Mn}$ & 3.49 & 8.71 & 4.74 \\
$\mathrm{Fe}$ & 40.84 & 36.66 & 38.29 \\
\hline
\end{tabular}

The experimental data were put into the above integral rate equations to evaluate the ratecontrolling step in the leaching process. The left equations were plotted with the leaching time, and the dependency of these models on the kinetic data was evaluated using correlation co-efficient values. The slopes of these plots were the apparent rate constants $\left(\mathrm{kr}_{\mathrm{r}}\right.$ and $\mathrm{kd}_{\mathrm{d}}$.

\subsubsection{Kinetics analysis of vanadium extraction}

The rate-determining step of the extraction of vanadium from the vanadium slag was studied, and the vanadium extraction rate at the temperature of $130{ }^{\circ} \mathrm{C}$ was fitted into the above equations, and the results were shown in Fig. 8. From Fig. 8, Eq. (7) fitted the experiment data perfectly with the $\mathrm{R}$ square correlation of 0.9956 , thus the kinetics of the vanadium extraction process was controlled by internal diffusion. The fitting of vanadium extraction rates over time at various temperatures were also performed, and the results were shown in Fig. 9 and Table 5. 


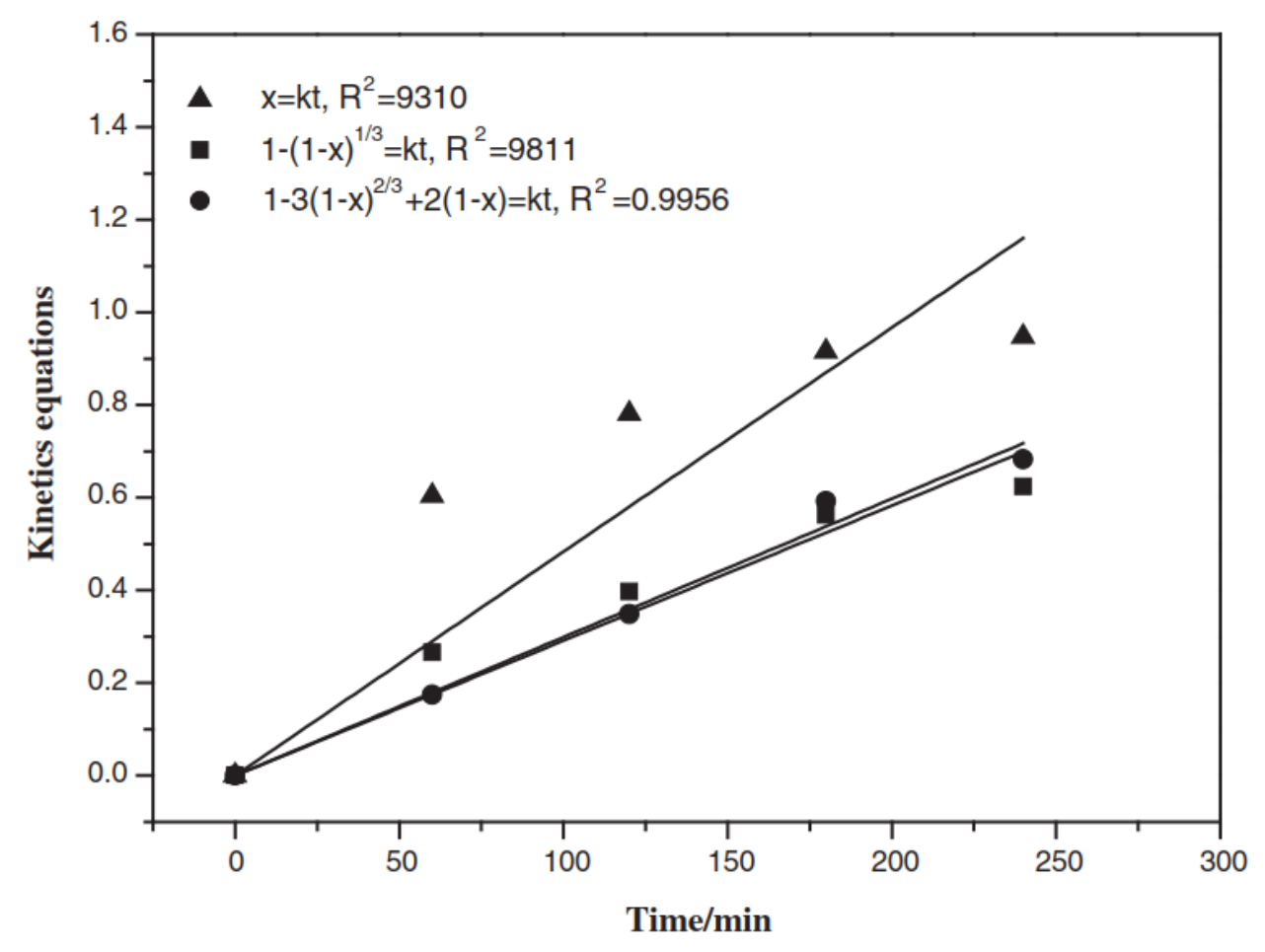

Fig. 8. Vanadium extraction kinetics with microporous filter at $130^{\circ} \mathrm{C}$.

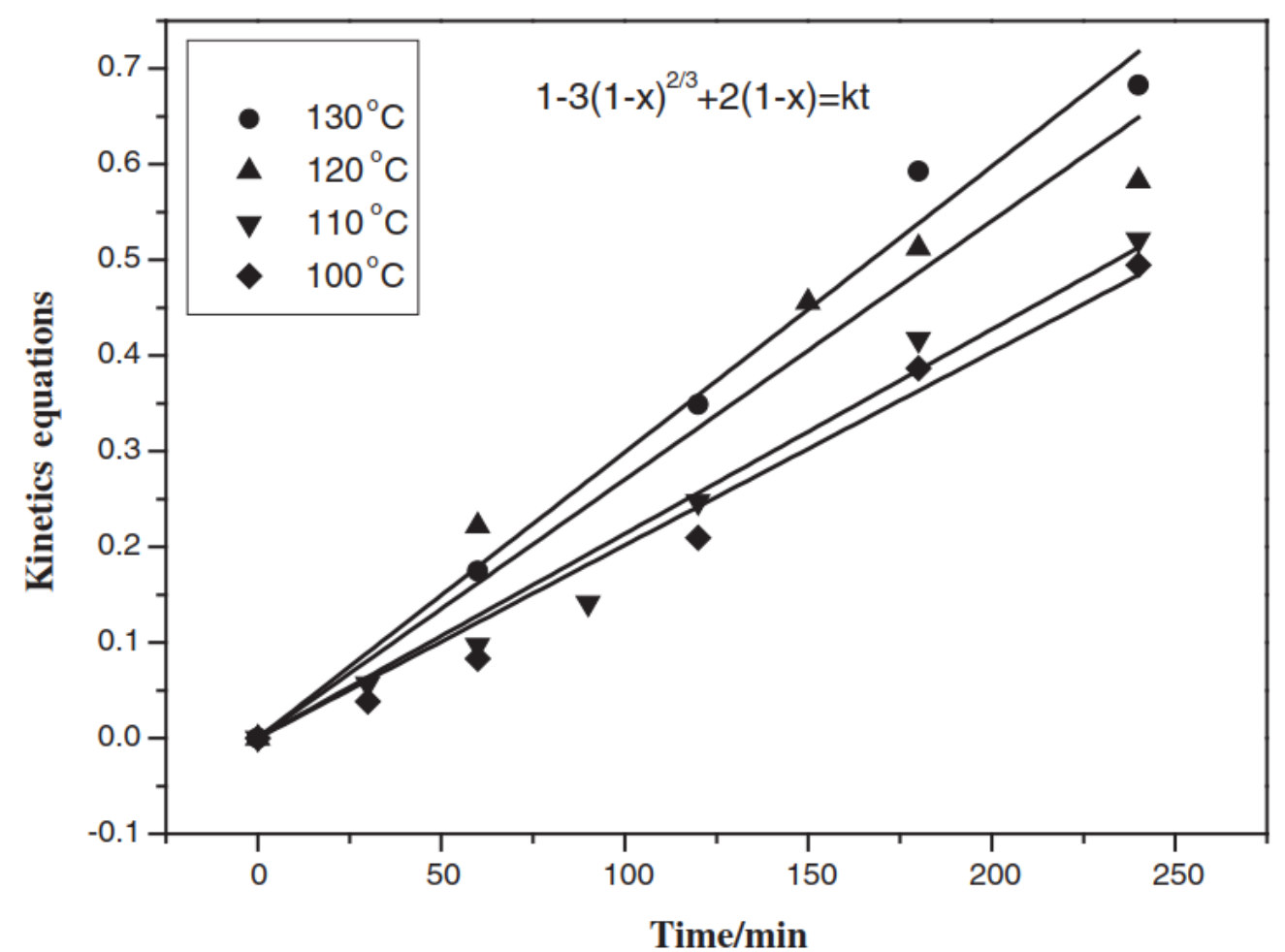

Fig. 9. Vanadium extraction kinetics with microporous filter at different temperatures. 
Table 5. Vanadium extraction kinetic parameters at different temperature.

\begin{tabular}{llll}
\hline Temperature $/{ }^{\circ} \mathrm{C}$ & $\mathrm{k}$ & $\mathrm{R}$ square correlation & Rate-determining step \\
\hline 100 & 0.00202 & 0.9920 & Internal diffusion \\
110 & 0.00214 & 0.9910 & Internal diffusion \\
120 & 0.00270 & 0.9870 & Internal diffusion \\
130 & 0.00299 & 0.9956 & Internal diffusion \\
\hline
\end{tabular}

It can be seen from the Table 5 that the reaction rate constant $k$ increases with an increase in the reaction temperature, and the vanadium extraction process was governed by internal diffusion at various temperatures, and therefore the vanadium extraction process could be further optimized by adjusting the mass transfer. The oxygen mass transfer improvement can apparently promote the vanadium extraction with the microporous filter application.

The apparent activation energy for vanadium extraction from the vanadium efficiency slag can be calculated using the Arrhenius equation based on the analysis, and the result was summarized in Fig. 10

$$
\operatorname{Ink}=\operatorname{In} A-\frac{E_{a}}{R} \times \frac{1}{T}
$$

where $\mathrm{k}, \mathrm{Ea}, \mathrm{A}, \mathrm{R}$, and $\mathrm{T}$ are respectively the kinetics constant, the apparent activation energy, the pre-exponential factor, the molar gas constant, and the Kelvin temperature.

From Fig. 10, the apparent activation energy for vanadium extraction from the vanadium slag was determined to be $17.57 \mathrm{~kJ} / \mathrm{mol}$, and the vanadium extraction kinetics equation could be obtained by combing Eq. 7 and 8, as expressed by Eq. (9).

$$
1+2(1-x)-3(1-x)^{2 / 3}=\exp (-0.567-2113.49 / T) \times \mathrm{t}
$$




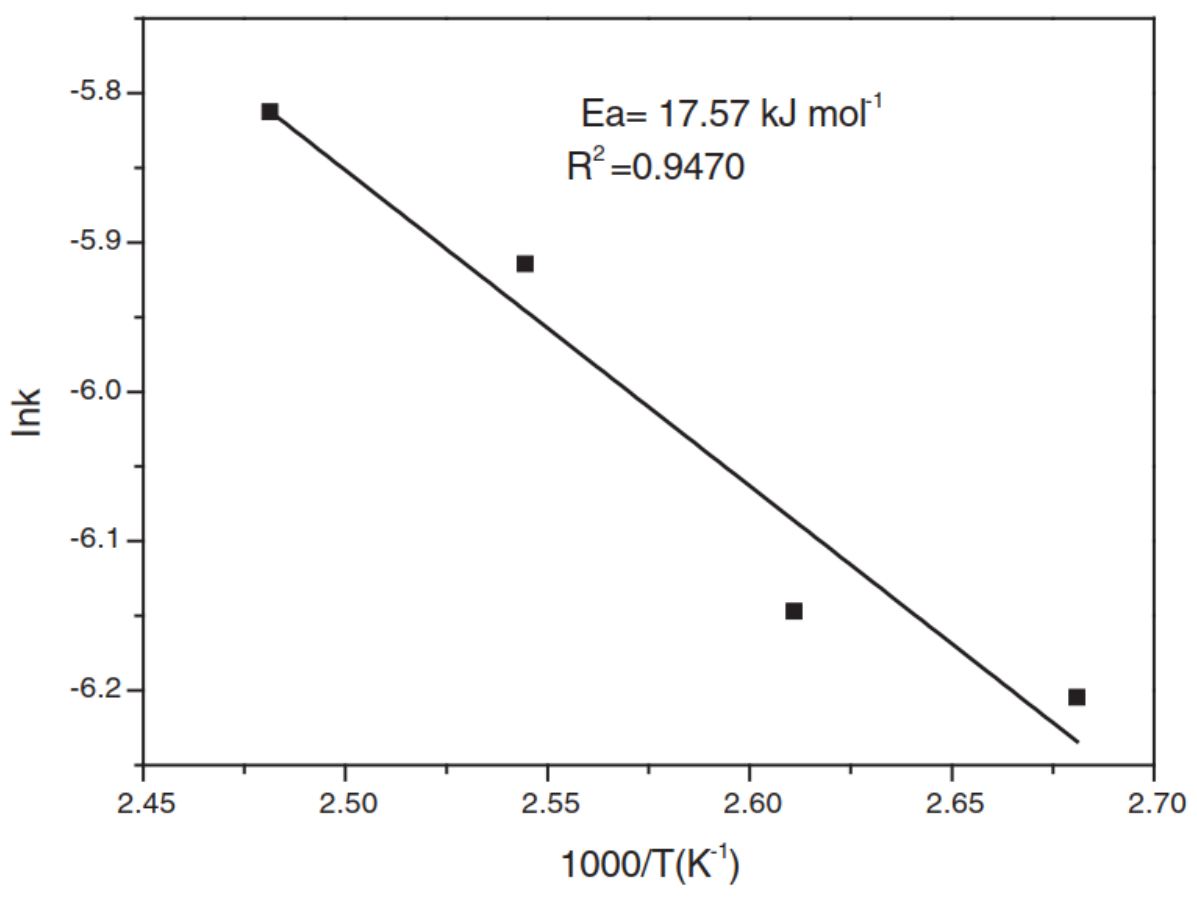

Fig. 10. Arrhenius plot for the leaching process of vanadium slag with microporous filter.

\section{Conclusion}

A microporous filter introduced aeration system was applied in processing the vanadium slag in concentrated $\mathrm{NaOH}$ solution. The oxygen mass transfer in alkaline concentration was studied, and it was discovered that the oxygen mass transfer coefficient was decreased with the alkaline concentration increased. Meanwhile, the microporous filter applied can improve the oxygen mass transfer coefficient greatly, especially in the high alkaline concentration. The reason for this is that the microporous filter can generate microbubbles, and the oxygen mass transfer of microbubbles are enhanced by the self-compression and shrinking.

To determine the best reaction conditions, various reaction parameters such as pore size of the microporous filter, alkali concentration, reaction temperature, the alkali to ore mass ratio and the stirring speed were studied, and it was discovered that under the best reaction conditions of alkali concentration of $60 \mathrm{wt} \%$, reaction temperature of $130{ }^{\circ} \mathrm{C}$, alkali to ore mass ratio of $6: 1$, stirring speed of $500 \mathrm{rpm}$, the extraction rate of vanadium could reach $97.41 \%$ after reacting for $6 \mathrm{~h}$. It was further observed that the extraction rates of vanadium showed an analogously parabolic characteristic with the increase in alkali concentration and the temperature, and increased with the alkali to ore mass ratio and stirring speed.

The SEM results indicated that the leaching process followed a shrinking core model. Kinetics calculation was studied. For the vanadium extraction process, the rate determining step could be controlled by internal diffusion and the apparent activation was calculated to 
be $17.57 \mathrm{~kJ} / \mathrm{mol}$, which was much lower than the previous reports in the literatures. The application of microporous filter for aeration in sub-molten solution could clearly improve the oxygen mass transfer and increase the vanadium extraction efficiency based on our work.

\section{Acknowledgements}

The authors gratefully acknowledge the financial support from the Major State Basic Research Development Program of China (973 program) under grant No. 2013CB632601, National Natural Science Foundation of China under Grant Nos. 91634111 and 51404227.

\section{References}

Agarwal, A., Ng, W.J., Liu, Y., 2011. Principle and applications of microbubble and nanobubble technology for water treatment. Chemosphere 84 (9), 1175-1180.

ASCE, 1997. Standard Guidelines for in Process Oxygen Transfer Testing, New York (18 pp.).

Bangalore, D.V., Bellmer, D.D., 2006. Microbubbles for enhancement of oxygen transfer in xanthan gum fermentation. Chem. Eng. Commun. 193 (10), 1232-1252.

Burns, S.E., Yiacoumi, S., Tsouris, C., 1997. Microbubble generation for environmental and industrial separations. Sep. Purif. Technol. 11, 221-232.

Chen, G., et al., 2013. An investigation on the kinetics of chromium dissolution from Philippine chromite ore at high oxygen pressure in $\mathrm{KOH}$ sub-molten salt solution. Hydrometallurgy 139, 46-53.

Chern, J.-M., Chou, S.-R., Shang, C.-S., 2001. Effects of impurities on oxygen transfer rates in diffused aeration systems. Water Res. 35 (13), 3041-3048.

Chu, L.-B., et al., 2007. Enhanced ozonation of simulated dyestuff wastewater by microbubbles. Chemosphere 68 (10), 1854-1860.

Chu, L.-B., Xing, X.-H., Yu, A.-F., Sun, X.-L., Jurcik, B., 2008. Enhanced treatment of practical textile wastewater by microbubble ozonation. Process. Saf. Environ. Prot. 86 (5), 389-393.

Gurol, M.D., Nekouinaini, S., 1985. Effect of organic substances on mass transfer in bubble aeration. J. Water Pollut. Control Fed. 57 (3), 235-240.

Koide, K., Hayashi, T., Sumino, K., Iwamoto, S., 1976. Mass transfer from single bubbles in aqueous solutions of surfactants. Chem. Eng. Sci. 31 (10), 963-967. 
Li, P., Takahashi, M., Chiba, K., 2009. Enhanced free-radical generation by shrinking microbubbles using a copper catalyst. Chemosphere 77 (8), 1157-1160.

$\mathrm{Li}, \mathrm{C}$., et al., 2011. Effect of oxidation on vanadium extraction from stone coal with calcified roasting. J. Cent. S. Univ. Sci. Technol. 42 (1), 7-10.

Li, H.-Y., Wang, K., Hua, W.-H., Yang, Z., Zhou, W., Xie, B., 2016. Selective leaching of vanadium in calcification-roasted vanadium slag by ammonium carbonate. Hydrometallurgy $160,18-25$.

Liu, S., et al., 2010. Effect of micro-bubbles on coagulation flotation process of dyeing wastewater. Sep. Purif. Technol. 71 (3), 337-346.

Liu, B., et al., 2012. The redox behavior of vanadium in alkaline solutions by cyclic voltammetry method. Electrochim. Acta 76, 262-269.

Liu, B., et al., 2013a. A novel method to extract vanadium and chromium from vanadium slag using molten NaOH-NaNO3 binary system. AICHE J. 59 (2), 541-552.

Liu, H.-B., et al., 2013b. Kinetics analysis of decomposition of vanadium slag by $\mathrm{KOH}$ submolten salt method. Trans. Nonferrous Metals Soc. China 23 (5), 1489-1500.

Loubière, K., Castaignède, V., Hébrard, G., Roustan, M., 2004. Bubble formation at a flexible orifice with liquid cross-flow. Chem. Eng. Process. Process Intensif. 43 (6), 717-725.

Maeda, Y., Hosokawa, S., Baba, Y., Tomiyama, A., Ito, Y., 2015. Generation mechanism of micro-bubbles in a pressurized dissolution method. Exp. Thermal Fluid Sci. 60, 201-207.

Mishima, K., et al., 1996. Measurement and correlation of solubilities of oxygen in aqueous solutions containing galactose and fructose. Fluid Phase Equilib. 118 (2), 221-226.

Mishima, K., et al., 1997. Measurement and correlation of solubilities of oxygen in aqueous solutions containing ribose and raffinose. Fluid Phase Equilib. 134 (1-2), 277-283.

Pitzer, K.S., 1973. Thermodynamics of electrolytes. I. Theoretical basis and general equations. J. Phys. Chem. 77 (2), 268-277.

Qiu, S., et al., 2011. Dissolution kinetics of vanadium trioxide at high pressure in sodium hydroxide-oxygen systems. Hydrometallurgy 105 (3-4), 350-354. 
Sun, Z., Zhang, Y., Zheng, S.-L., Zhang, Y., 2009. A new method of potassium chromate production from chromite and $\mathrm{KOH}-\mathrm{KNO} 3-\mathrm{H} 2 \mathrm{O}$ binary submolten salt system. AICHE J.55 (10), 2646-2656.

Takahashi, M., et al., 2003. Effect of shrinking microbubble on gas hydrate formation.

J. Phys. Chem. B 107 (10), 2171-2173.

Takahashi, M., Chiba, K., Li, P., 2007. Free-radical generation from collapsing microbubbles in the absence of a dynamic stimulus. J. Phys. Chem. B 111 (6), 1343-1347.

Terasaka, K., Hirabayashi, A., Nishino, T., Fujioka, S., Kobayashi, D., 2011. Development of microbubble aerator for waste water treatment using aerobic activated sludge. Chem. Eng. Sci. 66 (14), 3172-3179.

Tsang, Y.H., Koh, Y.H., Koch, D.L., 2004. Bubble-size dependence of the critical electrolyte concentration for inhibition of coalescence. J. Colloid Interface Sci. 275 (1), 290-297.

Voglauer, B., Grausam, A., Jörgl, H.P., 2004. Reaction-kinetics of the vanadium roast process using steel slag as a secondary raw material. Miner. Eng. 17 (2), 317-321.

Wang, Z.-H., et al., 2014. Research and prospect on extraction of vanadium from vanadium slag by liquid oxidation technologies. Trans. Nonferrous Metals Soc. China 24 (5), 1273-1288.

Wang, Z., et al., 2015. Electrochemical decomposition of vanadium slag in concentrated $\mathrm{NaOH}$ solution. Hydrometallurgy 151, 51-55.

Yang, Z., Li, H.-Y., Yin, X.-C., Yan, Z.-M., Yan, X.-M., Xie, B., 2014. Leaching kinetics of calcification roasted vanadium slag with high $\mathrm{CaO}$ content by sulfuric acid. Int. J. Miner.Process. 133, 105-111.

Zhang, Y., Zheng, S.-L., Xu, H.-B., Du, H., Zhang, Y., 2010. Decomposition of chromite ore by oxygen in molten $\mathrm{NaOH}-\mathrm{NaNO} 3$. Int. J. Miner. Process. 95 (1-4), 10-17.

Zhang, J., Zhang, W., Zhang, L., Gu, S., 2015. Mechanism of vanadium slag roasting with calcium oxide. Int. J. Miner. Process. 138, 20-29. 